DOI: $10.34185 / 1991-7848 . i t m m .2020 .01 .033$

\title{
SIMPLE DYNAMIC LOSS-MINIMIZING FLUX LEVEL CONTROL OF FIELD-ORIENTATION INDUCTION MOTOR DRIVES
}

\author{
Diachenko Hryhorii, Aziukovskyi Oleksandr \\ Dnipro University of Technology, Ukraine
}

\begin{abstract}
The current paper discusses the adaptive choice of a filter time constant for filtering the steady-state flux reference in the energyefficient control problem of field-orientation induction machines in transient behavior when load and speed conditions are changing taking into account the effect of the main induction saturation. It is shown that by appropriately managing the flux linkage rate of change the energy losses per full operation cycle under torque changes can be significantly reduced compared to the conventional cases. The analysis for the appropriate choice of the filter time constant as a fraction of the rotor time constant is based on a numerical study and simulation results for three different induction machines with different rated powers.
\end{abstract}

Keywords: ENERGY EFFICIENCY, POWER LOSS, FILTERING, ADAPTIVE SEARCH, DYNAMIC OPERATION.

The induction machine is widely used in industrial applications due to its robustness and its low cost compared to permanent magnet synchronous machines. However, in part-load operation, the efficiency of the induction machine dramatically decreases when the flux is kept at the nominal level. To address this issue different strategies have been developed in the past to increase the efficiency of the induction machine in a large operation range [1]. The main idea of these methods is to choose an appropriate value for the rotor flux depending on the machine load in a stationary state of the machine. This covers a large part of applications like pumps, fans or conveyor applications. However, when the induction machine is operated under changing loads these methods will not yield maximum efficiency.

The motivation of this paper is to give a simple and easy implementable solution to the problem of energy consumption minimization, which is characterized by a continuity of action, converges to the optimal flux level in the steady-state and 
expresses a compromise approach to the problem with changing loads in dynamics as well as provide an alternative solution to model predictive control [2].

The paper [3] addresses the matter, however, although the approach is in principle considered, neither adaptive search of time constant nor main inductance saturation are given.

It was noticed that the field regulator attempts to establish a new steady-state optimal value for the rotor flux linkage as quickly as possible and as a result uses a high magnitude of the field-producing current and reaches its output almost in no time. This is the main contribution to short-term high losses according to the stepwise approach denoted in Fig. 1a. This fact means that it is not profitable to use solely the conventional flux controller in dynamic mode.
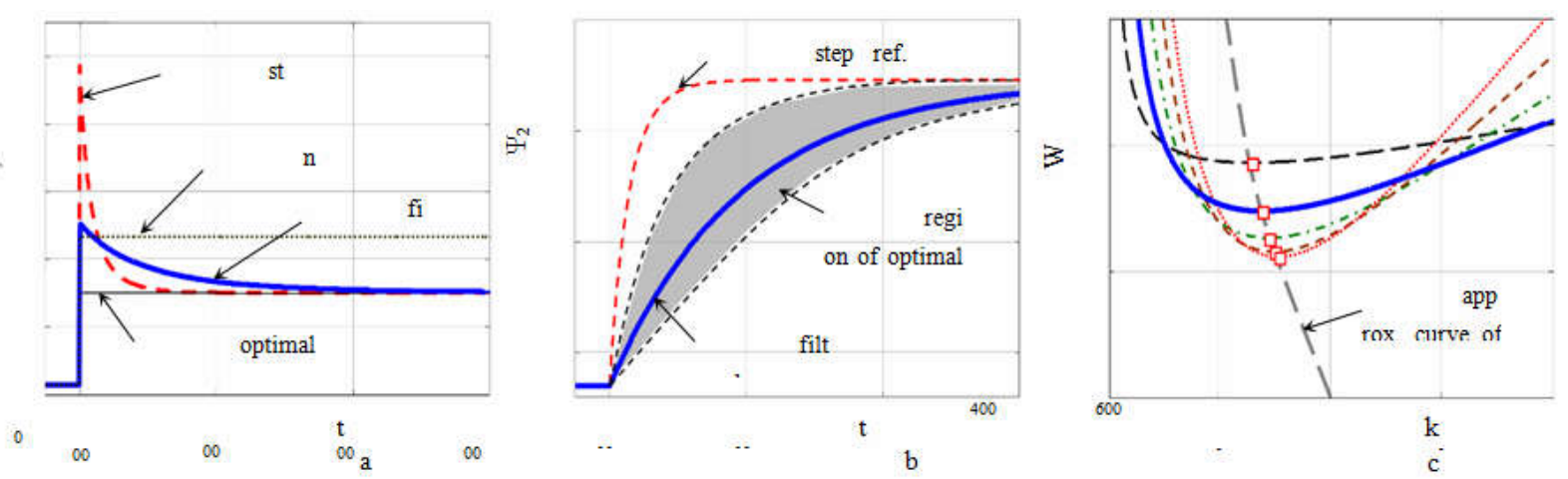

Fig. 1: a - power losses plot; $\mathrm{b}$ - rotor flux linkage trajectories;

c - energy loss trajectories for a certain load changes

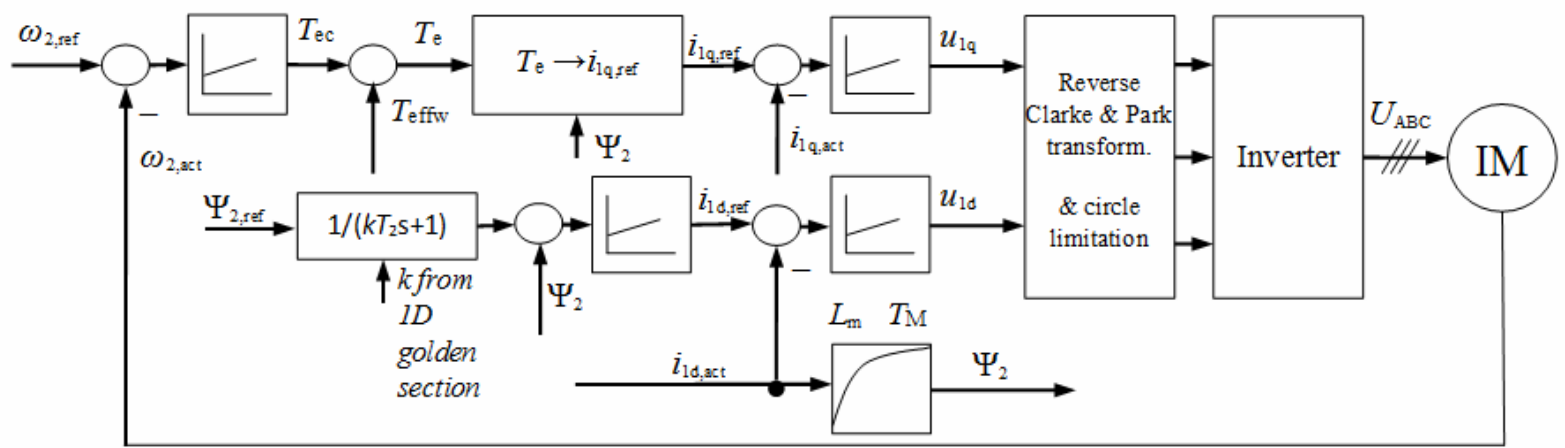

Fig. 2 - Control structure

Due to high instantaneous power loss overshoots under changing torques and, in addition, it leads to the increase of the total energy consumed per duty cycle.

The main idea of the proposed method is to combine approaches stepwise and nominal using low-pass filtering of the flux linkage reference, implemented by a first-order system in Fig. 2. The dependence of the total energy loss during the transition process from one steady-state point to another point was established as a 
function of the multiplying factor $k$ of the rotor time constant of an induction machine. The numerical investigation for three different induction machines with different rated powers has shown that the optimal value of the multiplier $k$ is in a range between 0.5 and 1 (example in Fig. 1c). In practice, the modeling of an induction machine as a linear object is inadequate even for nominal operating conditions because of magnetic saturation. In addition, the rotor time constant and sequentially the transient period is dependent on the main inductance value. In this regard, a flux observer, as well as the main induction saturation function as a polynomial approximation of the experimentally measured data points, are used in the control structure. Moreover, in contrast to [3] where factor $k$ had fixed value over wide operation range, it was suggested to effectively search the minimum point of the loss trajectories (Fig. 1c) by means of a 1-D search method. At each sampling period, the golden section method is applied to search the loss-minimizing factor $k$ as a fraction of the rotor time constant.

This approach reduces the peak power loss in case of a change in load or speed ramp and simultaneously converges to the optimal value of magnetic flux linkage in the steady-state mode of operation. Fig. 1a illustrates the proposed strategy.

The advantage of the proposed approach is that it is simple enough to implement, the resulting optimal trajectory is close to the exact solution, and most importantly, the same algorithm is used both to minimize power in statics and dynamics when load and speed conditions are changing.

\section{References}

1. Bazzi, A., \& Krein, P. (2009). A Survey of real-time power-loss minimizers for induction motors. IEEE Electric Ship Technologies Symposium, ESTS 2009. (pp. 98 - 106).

DOI: 10.1109/ESTS.2009.4906500.

2. Diachenko, G., Aziukovskyi, O., Rogoza, M., \& Yakimets, S. (2019). Optimal field-oriented control of an induction motor for loss minimization in dynamic operation. 2019 International Conference on Modern Electrical and Energy Systems (MEES), (pp. 94-97). Kremenchuk, Ukraine. DOI: 10.1109/MEES.2019.8896455.

3. Diachenko, G., \& Schullerus, G. (2015). Simple dynamic energy efficient field oriented control in induction motors. Proceeding of the 18th International Symposium on Power Electronics. Novi Sad, Serbia. 